\title{
EFFECTS OF EXERGAMES ON BALANCE, FUNCTIONAL MOBILITY, AND QUALITY OF LIFE OF GERIATRICS VERSUS HOME EXERCISE PROGRAMME: RANDOMIZED CONTROLLED STUDY
}

\author{
Ali Yavuz Karahan ${ }^{1}$, Fatih Tok², Halil Taşkın ${ }^{3}$, Seher Küçüksaraç ${ }^{1}$, Aynur Başaran ${ }^{1}$, Pelin Yıldırım ${ }^{4}$ \\ ${ }^{1}$ Department of Physical Medicine and Rehabilitation, Beyhekim State Hospital of Konya, Turkey \\ ${ }^{2}$ Iskenderun Marine Regiment Medical Center, Iskenderun, Hatay, Turkey \\ ${ }^{3}$ Department of Coaching Education Programme, School of Physical Education and Sports, Selcuk University of Konya, Turkey \\ ${ }^{4}$ Department of Physical Medicine and Rehabilitation, Derince Education and Training Hospital, Kocaelei, Turkey
}

\begin{abstract}
SUMMARY
Aim: To compare the effects of exergames (EGs) using the Xbox KinectTM device and home exercise (HE) on balance, functional mobility, and quality of life of individuals aged 65 years or older.

Method: One hundred participants who met the inclusion criteria were randomized to the EG or HE group. The EG group took part in a 6-week programme using the Xbox360Kinect ${ }^{\mathrm{TM}}$ device, and the HE group took part in a 6-week balance exercise programme at home 5 days a week. The Berg Balance Scale (BBS) was used to assess balance, the Timed Up and Go (TUG) test was used to evaluate functional walking, and the Short Form 36 (SF-36) was used to assess quality of life.

Results: Forty-eight participants in the EG group and 42 participants in the HE group completed the study. The groups were similar in terms of age, sex, and pretreatment values of BBS, TUG, and SF-36. Although the BBS scores of both groups improved significantly $(a l l ~ p<0.05)$, the post-treatment scores of the EG group were better than those of the HE group. The TUG scores improved only in the EG group $(p<0.05)$. The increase in the BBS scores and decrease in the TUG test scores were significant only in the EG group (all $p<0.05$ ). A significant improvement was also observed in the quality of life parameters of physical functioning, social role functioning, physical role restriction, general health perceptions, and physical component scores in the post-exercise evaluations of the EG group. The participants commented that they found the EG programme very entertaining.

Conclusion: The EG can be considered a safe, entertaining and sustainable alternative to $\mathrm{HE}$ programmes, and it may have positive effects on balance, functional walking and quality of life in geriatric subjects.
\end{abstract}

Key words: exergames, geriatrics, balance, walking, quality of life

Address for correspondence: A. Y. Karahan, Department of Physical Medicine and Rehabilitation, Beyhekim State Hospital, Konya, Turkey. E-mail: ayk222@hotmail.com.

\section{INTRODUCTION}

Today, many children and adults use videogames as recreational tools. Many of these require physical activity for playability. Many gamers are particularly interested in games where they have to move part or all of their bodies to control an avatar or activity during the game (1). Although such games are limited to the indoor area and a television game console, they are predicted to play a major role in daily physical activity recommended for children and young people (2). Even though the primary purpose of video game developers is to make the games more enjoyable, these games have positive impacts on physical activity and therefore health. In particular, after 2000, the academic interest in this topic increased when games requiring physical activity were shown to be effective in increasing required daily energy expenditure levels (2). In 2007, "exergames" (the term derived from "exercise" and "game") were introduced to the
English scientific literature (2). The popularity of rehabilitation programmes based on virtual reality has also increased, with EGs included or adapted for use in such programmes, especially in obesity prevention programmes and hemiplegia rehabilitation in children (1-3). Dancing, calisthenics, activities with exercisers, aqua therapy, and group exercises have been reported to increase exercise motivation among the geriatric population $(4,5)$. The use of virtual reality programmes in geriatric rehabilitation has also attracted attention. However, there are only a few studies on the use of EGs for exercising in older people, with researchers reporting positive effects of EGs with the Nintendo Wii Fit ${ }^{\mathrm{TM}}$ game console on the balance of geriatric patients (6-9).

Accordingly, the aim of this study was (1) to demonstrate the effects of EG using the Xbox 360 Kinect $^{\mathrm{TM}}$ game console on balance, functional mobility, and quality of life of geriatrics and (2) to compare the effects of EG with those achieved in the home exercise (HE) programme. 


\section{MATERIALS AND METHODS}

One hundred participants aged 65 years or older who attended the Physical and Rehabilitation Medicine outpatient clinic from September 2013 to November 2013 were included in the study. All subjects were informed about the content, purpose and execution of the study, and all completed informed consent forms. The local ethics committee approved the study. The inclusion criteria were as follows:

- Individuals who could cooperate, as assessed by the Mini Mental Status test (those with a score of 25 or higher were included in the study);

- Individuals who could ambulate without support;

- Individuals with a score of 3 or lower on the Visual Analogue Scale evaluation for pain in any part of their bodies;

- Individuals who did not exercise regularly. The exclusion criteria were as follows:

- The presence of cardiopulmonary dysfunction that would hinder aerobic exercising (e.g., acute congestive heart failure, unstable angina pectoris, or third-stage cardiac block);

- The presence of central or peripheral neurological disease;

- The presence of problems that hindered standing (e.g., previous lower extremity surgery or lower extremity pain);

- A diagnosis of a serious psychiatric disorder, serious hearing disorder or serious visual disorder.

One hundred patients who met the inclusion criteria were divided into the EG group and HE group using the complete randomization method (coin flipping). The randomized assignment with even chances resulted in 54 cases in the EG group and 46 cases in the HE group. The study progression is outlined in the flow diagram (Fig. 1).

The HE group undertook balance exercises, including stretching exercises for the hamstring, quadriceps, pelvic girdle, and pectoral group muscles, and strengthening exercises for large muscle groups (quadriceps, hamstring, biceps, and abdominal muscles). These included standing on two feet, a semitandem

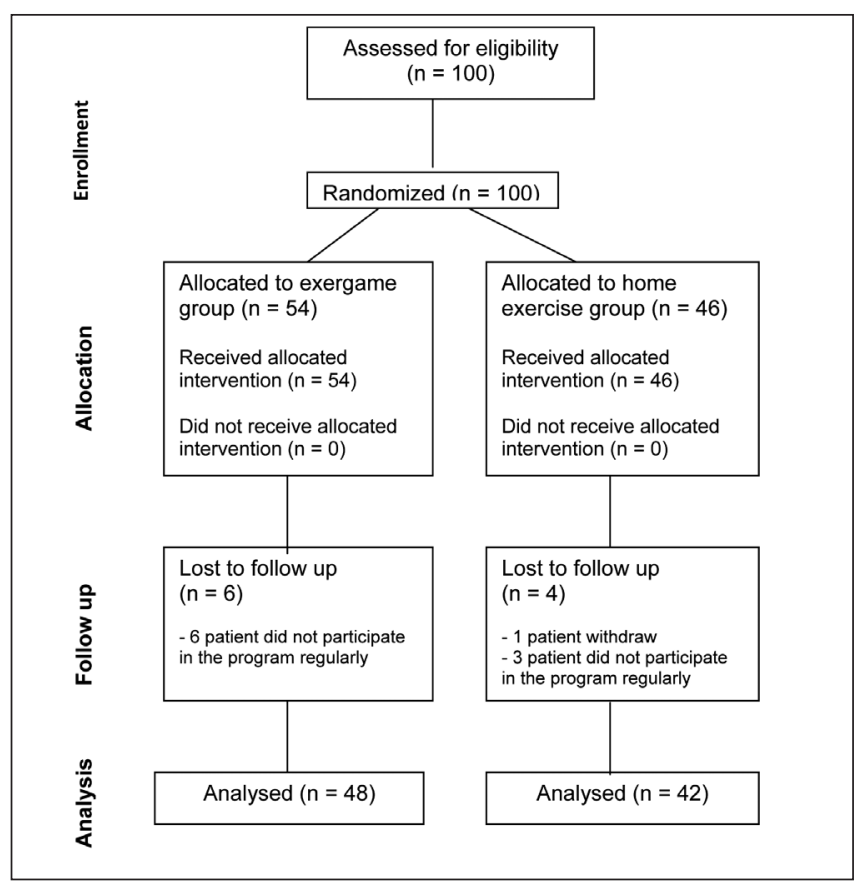

Fig. 1. Flow diagram. stance, standing on one foot, standing on heels, and standing on toes. Physicians ensured that the subjects performed the exercises correctly. The subjects were instructed to perform the exercises for at least $30 \mathrm{~min}$, at least 5 days a week, for 6 weeks for a total of 30 exercise sessions. They were advised to discontinue the exercises and to consult a physician in case of warning symptoms, such as chest pain, dizziness or palpitation during the exercises.

For the EG group, a game set comprising the Xbox 360 Kinect game console and 46-inch LCD TV was placed in a $20 \mathrm{~m}^{2}$ room. The group was provided with video games. These consisted of "Kinect Adventures, Kinect Sports, and Kinect Sports Season two" programmes, which included football, tennis, table tennis, skiing, golf, volleyball, and bowling game simulations. Physicians described the games to the subjects. Each subject played the games for 30 min, 5 days a week, for 6 weeks (30 sessions in total) in the company of an experienced nurse who was able to perform cardiopulmonary follow-up (Fig. 2).

The Berg Balance Scale (BBS), Timed Up and Go (TUG) Test, and Short Form 36 (SF-36) were administered before and immediately after the programme to evaluate balance, functional mobility, and health quality of life. After the programme, the participants were also questioned about the enjoyment levels of various exercises.

\section{Berg Balance Scale}

The Berg Balance Scale is considered the gold standard for functional evaluation of balance. It evaluates the capability of a person to maintain a static position during 14 different activities (10). The participant is evaluated during each activity by an observer and scored on a scale from 1 to 4 . The highest total score is 56 for rapid, flowing and easily performed activities. Scores between 0 and 20 indicate balance impairment, between 21 and 40 denote acceptable balance, and between 41 and 60 denote good balance $(10,11)$.

\section{Timed Up and Go Test}

The Timed Up and Go test is an objective, reliable and simple scale for evaluating functional mobility. It can also be used to predict the risk of falls. In the test, the person is asked to stand up off a seat, walk $3 \mathrm{~m}$, turn around, return to the seat, and sit. The

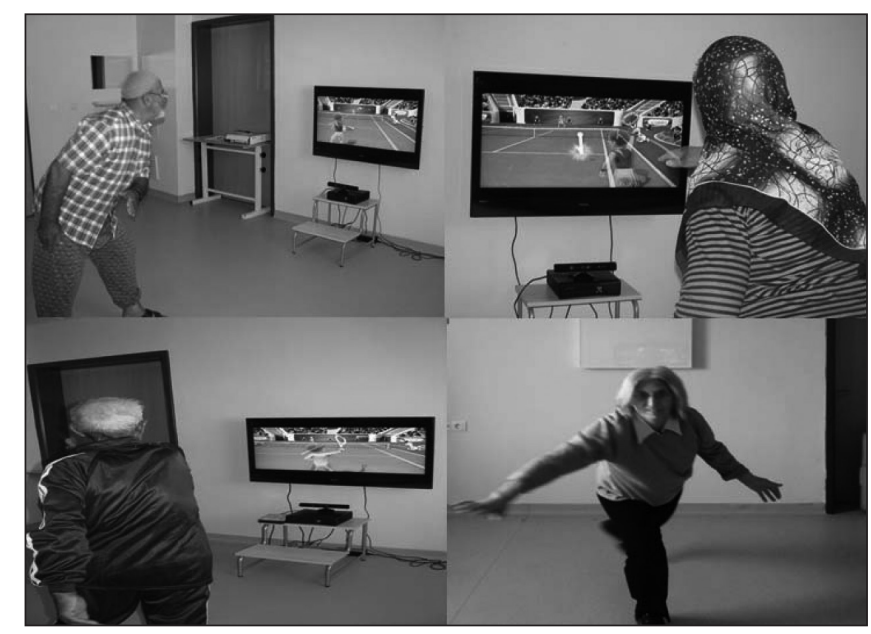

Fig. 2. Participants while exergaming. 
score is calculated by measuring the time of test completion. The use of walking aids is allowed during the test $(12,13)$.

\section{Short Form 36}

The SF-36 form is a patient-reported survey of patient health. It is composed of eight subscales and 36 items, which evaluate physical and mental health (14). The subscales are physical functioning, physical role restriction, bodily pain, general health perceptions, vitality, social role functioning, emotional role restriction, and mental health. It has been adapted to Turkish society (14). Scores vary between 0 and 100 , with 100 points denoting the best state of health, and 0 denoting the worst state. Normal data of the general population were calculated using a special calculation method, and the Physical Component Summary was calculated using the first four subscale scores. The Mental Component Summary was calculated with the last four subscales. Thus, the physical and mental quality of life scores were calculated $(14,15)$.

\section{Exercise Enjoyment}

A 5-point Likert-type scale was used to measure the enjoyment of exercises. The subjects who participated in the exercise programmes were asked to respond to the following question: "The exercise programme I participated in was poor, fair, average, good, or excellent,” with 1, 2, 3, 4, and 5 points awarded, respectively.

\section{Statistical Evaluation}

The SPSS for Windows 10.0 (IBM, Inc., USA) software package was used for the statistical evaluation of the data. The conformity of continuous variables with a normal distribution was investigated using the Kolmogorov-Smirnov test. All variables were distributed normally. Nominal data were assessed with the Pearson's ChiSquare (with Yates' correction) test. The paired-samples T-test was used to compare the intragroup pre- and post-exercise values. The independent-samples T-test was used for intergroup comparisons. The level of significance was set at $\mathrm{p}<0.05$.

\section{RESULTS}

Data from 42 participants in the HE group and 48 participants in the EG group who completed the 30 -session exercise programme were evaluated in our study. The mean age was 71.5 years in the HE group and 71.3 years in the EG group. The demographic data of the groups are shown in Table 1 . The groups were similar in terms of age, gender, body-mass index, and Mini Mental Test scores (all p>0.05) (Table 1).

Although the BBS scores of both groups improved significantly (all $\mathrm{p}<0.05)$, the TUG scores improved only in the EG group $(p<0.05)$. The post-treatment scores of the EG group were better than those of the HE group. However, the increase in the BBS scores and decrease in the TUG test scores were significant only in the EG group (all $\mathrm{p}<0.05$ ) (Table 2). No statistically significant

Table 1. Demographics of the groups

\begin{tabular}{|l|c|c|c|}
\hline & $\begin{array}{c}\text { Home exercise group } \\
(\mathrm{n}=42)\end{array}$ & $\begin{array}{c}\text { Exergame group } \\
(\mathrm{n}=48)\end{array}$ & $\mathrm{p}$ \\
\hline Age (year) & $71.5 \pm 4.7$ & $71.3 \pm 6.1$ & 0.97 \\
\hline Gender & $18(42.8 \%)$ & $21(43.8 \%)$ & \multirow{2}{*}{0.12} \\
\hline Women $\mathrm{n}(\%)$ & $24(57.2 \%)$ & $27(56.2 \%)$ & 0.75 \\
\hline Men $\mathrm{n}(\%)$ & $27.2 \pm 3.2$ & $26.8 \pm 4.6$ & 0.96 \\
\hline Body Mass Index $\left(\mathrm{kg} / \mathrm{m}^{2}\right)$ & $28.1 \pm 2.2$ & $28.0 \pm 2.4$ & \\
\hline Mini Mental Test Score & & & \\
\hline
\end{tabular}

Table 2. Assessment of balance and functional mobility parameters

\begin{tabular}{|c|c|c|c|c|c|}
\hline & \multicolumn{2}{|c|}{$\begin{array}{l}\text { Home exercise group } \\
\qquad(n=42)\end{array}$} & \multicolumn{2}{|c|}{$\begin{array}{l}\text { Exergame group } \\
\qquad(n=48)\end{array}$} & \multirow{2}{*}{$\begin{array}{c}\text { HE group vs. } \\
\text { EG group } \\
p\end{array}$} \\
\hline & Mean \pm SD & $\mathrm{p}$ & Mean \pm SD & $\mathrm{p}$ & \\
\hline \multicolumn{6}{|l|}{ Berg Balance Scale } \\
\hline Baseline & $49.42 \pm 3.79$ & \multirow{3}{*}{$<0.001^{\star}$} & $49.85 \pm 3.88$ & \multirow{3}{*}{$<0.001^{*}$} & 0.760 \\
\hline After treatment & $51.16 \pm 4.14$ & & $54.91 \pm 2.67$ & & $<0.001$ \\
\hline Amount of change & 1.74 & & 5.06 & & 0.020 \\
\hline \multicolumn{6}{|c|}{ Time Up and Go Test (sn) } \\
\hline Baseline & $8.69 \pm 1.70$ & \multirow{3}{*}{$0.711^{\star}$} & $8.70 \pm 1.71$ & \multirow{3}{*}{$<0.001^{\star}$} & 0.811 \\
\hline After treatment & $8.60 \pm 1.83$ & & $8.04 \pm 16.47$ & & 0.090 \\
\hline Amount of change & 0.9 & & 0.64 & & 0.020 \\
\hline
\end{tabular}

*Baseline versus after treatment

$\mathrm{HE}$ - Home exercise, EG - Exergame 
improvement was observed in the SF-36 parameters following the exercise programme in the HE group. A statistically significant improvement was observed in the quality of life parameters of physical functioning, social role functioning, physical role restriction, general health perceptions, and physical component scores in the post-exercise evaluations of the EG group (all $\mathrm{p}<0.05$ ). However, the pre- and post-treatment parameters of the SF-36 were similar between the two groups (all $\mathrm{p}>0.05$ ).

The post-treatment assessment of exercise participation motivation in the HE group with a 5-point Likert scale revealed that $7.1 \%(n=3)$ of the participants evaluated the study as "not pleasant at all”, $16.6 \%(n=7)$ rated it as "unpleasant”, 42.8\% $(n=18)$ rated it as "moderately pleasant", $21.4 \%(n=9)$ rated it as "fairly pleasant”, and $11.9 \%(n=5)$ rated it as “very pleasant”. In the EG group, $18.7 \%(\mathrm{n}=9)$ of the participants evaluated the study as "moderately pleasant", $37.5 \%(n=18)$ considered it "fairly pleasant”, and 43.7\% $(n=21)$ considered it "very pleasant" (Fig. 3).

\section{DISCUSSION}

In this study, we observed that EG had positive effects on balance, functional walking, and quality of life of geriatric subjects and that they regarded the programme as entertaining and sustainable.

Motion sensors are used in video games to draw more attention to the game. The ability of this mobility to counteract inertia has drawn significant attention from clinicians. Previous studies showed that EG had positive effects on physico-social parameters, such as cognitive skills, coordination, self-esteem, self-sufficiency, motivation, attention, and proprioception, especially among children and adolescent populations $(1,2)$.These studies also emphasized the safety and sustainability of EGs $(1,2)$.

Investigating the efficacy of EGs as an alternative exercise method in the geriatric population is quite difficult because, in general, they do not exercise regularly (7-9). Franco et al. (16) compared the efficiency of EG with Wii Fit ${ }^{\mathrm{TM}}$ with HE and physiotherapist-guided balance exercises in a study of the geriatric population. Three groups, each comprising 11 patients, performed two exercises per week for 3 weeks. The researchers reported positive effects of the physiotherapist-guided balance exercises and EG. They also noted that EG was an entertaining alternative for older people. Szturm et al. (9) divided geriatric patients into

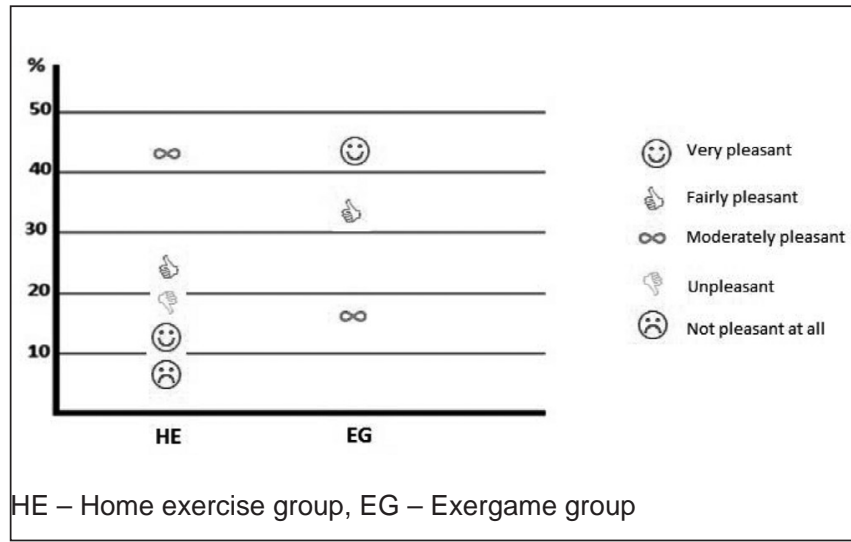

Fig. 3. Assessment of the enjoyment of the exercise. two groups of 15 who performed EG and dynamic balance exercises 45 min twice a week in 16 sessions. The follow-up revealed that the BBS parameters significantly improved. However, they found no significant improvements in the TUG test parameters of either group (9). The same study found that the instant feedback on the participant's performance in EG increased the person's motivation during EG (9). In our study, the BBS and TUG scores of the EG group significantly improved, in line with the findings of previous studies.

Agmon et al. (8) studied seven geriatric patients with BBS values less than 52 . They gave each participant a game console (Wii Fit), and the participants performed the EG application at home. After 50 exercise sessions, the BBS and walking speed of all participants increased. The same study reported that the participants considered the HE programme with their family members as sustainable and entertaining. Also all subjects in the EG group evaluated the exercise programme as pleasant, fairly pleasant, or very pleasant. This finding is compatible with that of our study. In the present study, $75.8 \%$ of the subjects in the HE group evaluated the exercise programme as pleasant, fairly pleasant or very pleasant. In contrast, $24.2 \%$ of the subjects in the HE group evaluated the programme as not pleasant at all or unpleasant. This difference between the two groups is most likely due to the entertaining and enjoyable nature of EGs.

Based on a review of 11 articles, Verheijden Klompstra et al. reported that EGs were a safe, easily applied, and sustainable method, with positive effects on balance, cognitive functions, depression, and quality of life (3). The main advantages of our study are the inclusion of "TUG parameters", which are the less sensitive to sample size than other types of tests, the large number of participants $(n=100)$, the high completion rate $(n=90)$, and the number of exercise sessions $(n=30$ sessions, 900 min in total). Other advantages are the inclusion of a control group of participants who received detailed descriptions of the exercises but who had the option to attend part (i.e., participation was not mandatory). In addition, a device (joystick) did not have to be used with the game console (Xbox360 Kinect ${ }^{\mathrm{TM}}$ ) in EG.

The limitations of the present study are the lack of standard exercise programme assignment in the exercise groups as well as the absence of objective measurement of muscle power in the follow-up assessments and the hospital-dependent setting of the exercises. Another limitation is the lack of exercise stress test before starting the exercise programmes. The absence of any observations of cardiopulmonary, locomotor or neurological complications during or after the exercises was interpreted as a sign of the EG safety. The results of the present study seem to be significant.

In conclusion, EG can be regarded as a safe, entertaining, and sustainable alternative exercise programme that may have positive effects on balance, functional walking and quality of life of geriatric subjects.

\section{Acknowledgement}

This study was presented at 7th Academic Geriatric Congress which was organized in Antalya, Turkey, May 28 - 31, 2014.

\section{Conflict of Interests}

None declared 


\section{Author Contributions}

Research concept and design: AYK; collection and/or assembly of data: AYK, FT, HT, SK, AB; data analysis and interpretation: AYK, FT, HT, SK, AB; writing the article: AYK, FT, PY; critical revision of the article: AYK, FT, HT, SK, AB; final approval of the article: AYK, FT, HT, SK, AB, PY; statistical analysis: AYK, FT.

\section{REFERENCES}

1. Staiano AE, Calvert SL. The promise of exergames as tools to measure physical health. Entertain Comput. 2011 Jan 1;2(1):17-21.

2. Staiano AE, Calvert SL. Exergames for physical education courses: physical, social, and cognitive benefits. Child Dev Perspect. 2011 Jun;5(2):938.

3. Verheijden Klompstra L, Jaarsma T, Strömberg A. Exergaming in older adults: a scoping review and implementation potential for patients with heart failure. Eur J Cardiovasc Nurs. 2014 Oct;13(5):388-98.

4. Savas S. Prescribing exercise for geriatric group. Turk J Geriatr. 2011;14(3):281-7. (In Turkish.)

5. Karan A. Exercise and sports in the elderly. Turk J Phys Med Rehabil. 2006;52:A53-6. (In Turkish.)

6. Bateni H. Changes in balance in older adults based on use of physical therapy vs the Wii Fit gaming system: a preliminary study. Physiotherapy. 2012 Sep;98(3):211-6.

7. Duclos C, Miéville C, Gagnon D, Leclerc C. Dynamic stability requirements during gait and standing exergames on the wii fit ${ }^{\circledR}$ system in the elderly. J Neuroeng Rehabil. 2012 May 20;9:28. doi:10.1186/1743-00039-28.

8. Agmon M, Perry CK, Phelan E, Demiris G, Nguyen HQ. A pilot study of Wii Fit exergames to improve balance in older adults. J Geriatr Phys Ther. 2011 Oct-Dec;34(4):161-7.
9. Szturm T, Betker AL, Moussavi Z, Desai A, Goodman V. Effects of an interactive computer game exercise regimen on balance impairment in frail community-dwelling older adults: a randomized controlled trial. Phys Ther. 2011 Oct;91(10):1449-62.

10. Berg K, Wood-Dauphine S, Williams JI, Gayton D. Measuring balance in the elderly: preliminary development of an instrument. Physiother Can. 1989;41(6):304-11.

11. Sahin F, Yilmaz F, Ozmaden A, Kotevolu N, Sahin T, Kuran B. Reliability and validity of the Turkish version of the Berg Balance Scale. J Geriatr Phys Ther. 2008;31(1):32-7.

12. Podsiadlo D, Richardson S. The timed "Up \& Go": a test of basic functional mobility for frail elderly persons. J Am Geriatr Soc. 1991 Feb;39(2):142-8.

13. Alkan H,Topuz O, Yildiz N, Alkan S, Sarsan A, Ardic F. Efficacy of homebased exercise program and postural biofeedback therapy in reducing risk of falling among osteoporotic women over 65 years of age. Turk J Geriatr. 2011;14(1):26-34. (In Turkish.)

14. Kocyigit H, Aydemir O, Fisek, G Olmez N, Memis A. Validity and reliability of Turkish version of short form 36: a study of patients with rheumatoid disorder. Ilaç ve Tedavi Dergisi. 1999;12:102-6. (In Turkish).

15. Thrane G, Joakimsen RM, Thornquist E. The association between timed up and go test and history of falls: the Tromsø study. BMC Geriatr. 2007 Jan 12;7:1.

16. Franco JR, Jacobs K, Inzerillo C, Kluzik J. The effect of the Nintendo Wii Fit and exercise in improving balance and quality of life in community dwelling elders. Technol Health Care. 2012;20(2):95-115.

Received August 28, 2013 Accepted in revised form December 24, 2014 